CARPATHIAN J. MATH.

Volume 37 (2021), No. 2,

Pages $227-234$
Online version at https : //www . carpathian. cunbm . utcluj. ro/

Print Edition: ISSN 1584 - 2851; Online Edition: ISSN 1843 - 4401

DOI: https://doi.org/10.37193/CJM.2021.02.09

Dedicated to Prof. Ioan A. Rus on the occasion of his $85^{\text {th }}$ anniversary

\title{
Implicit functional differential equations with linear modification of the argument, via weakly Picard operator theory
}

\author{
ANTON S. MureşAN and VioricA MureşAN
}

ABSTRACT. Let $\mathbf{K}:=\mathbf{R}$ or $\mathbf{C}, 0<\lambda<1$ and $f \in C\left([0, b] \times \mathbf{K}^{3}, \mathbf{K}\right)$.

In this paper we use the weakly Picard operator theory technique to study the following functional-differential equation

$$
y^{\prime}(x)=f\left(x, y(x), y^{\prime}(x), y(\lambda x)\right), x \in[0, b] .
$$

\section{INTRODUCTION}

The theory of functional-differential equations and of functional-integral equations are both active fields in mathematics.

Many problems from physics, chemistry, astronomy, biology, engineering, social sciences lead to mathematical models described by functional-differential and functionalintegral equations (see [7], [8], [10], [13], [15], [19], [21]). The theory of this kind of equations has developed very much.

For the monographs in this field we quote here [1]-[4], such as a large number of papers, which contain a lot of techniques, ideas and applications.

Let $\mathbf{K}:=\mathbf{R}$ or $\mathbf{C}, 0<\lambda<1$ and $f \in C\left([0, b] \times \mathbf{K}^{3}, \mathbf{K}\right)$.

In this paper we use the weakly Picard operator theory technique to study the following functional differential equation

$$
y^{\prime}(x)=f\left(x, y(x), y^{\prime}(x), y(\lambda x)\right), x \in[0, b] .
$$

We obtain existence, uniqueness and data dependence results for the solution.

\section{PRELIMINARIES}

2.1. Notations and terminology. Let $X$ be a nonempty set and $A: X \longrightarrow X$ an operator. We denote by $A^{0}:=1_{X}, A^{1}:=A, \ldots, A^{n+1}:=A \circ A^{n}, n \in \mathbb{N}$, the iterate operators of $A$. Also:

$$
\begin{aligned}
P(X) & :=\{Y \subset X \mid Y \neq \emptyset\}, \\
I(A) & : \quad=\{Y \in P(X) \mid A(Y) \subset Y\},
\end{aligned}
$$

the family of all nonempty invariant subsets of $A$,

$$
F_{A}=\{x \in X \mid A(x)=x\},
$$

the fixed point set of the operator $A$.

Received: 12.01.20121. In revised form: 15.04.2021. Accepted: 21.04.2021

2010 Mathematics Subject Classification. 34K12, 47H10, 47H09, 45D05.

Key words and phrases. Implicit functional differential equation, Volterra operator, existence, uniqueness, data dependence, fixed point, contraction, weakly Picard operator.

Corresponding author: Anton S. Mureşan; anton.muresan@econ.ubbcluj.ro 
Let $(X, \rightarrow)$ be an $L-$ space (see [14],[18]).

Following Rus I. A. ( [11], [14], [15]), we have:

Definition 2.1. $A$ is a Picard operator if there exists $x^{*} \in X$ such that

1) $F_{A}=\left\{x^{*}\right\}$;

2) the successive approximation sequence $\left(A^{n}\left(x_{0}\right)\right)_{n \in \mathbb{N}}$ converges to $x^{*}$, for all $x_{0} \in X$

Definition 2.2. $A$ is a weakly Picard operator if the sequence $\left(A^{n}\left(x_{0}\right)\right)_{n \in \mathbb{N}}$ converges for all $x_{0} \in X$ and the limit (which generally depends on $x_{0}$ ) is a fixed point of $A$.

Definition 2.3. If $A: X \rightarrow X$ is a weakly Picard operator then we define the operator $A^{\infty}$ as follows:

$$
A^{\infty}: X \rightarrow X, A^{\infty}(x):=\lim _{n \rightarrow \infty} A^{n}(x), \text { for all } x \in X .
$$

We remark that $A^{\infty}(X)=F_{A}$. So $A^{\infty}$ is an retract of $X$ on $F_{A}$.

2.2. Weakly Picard operators. We have the following theorems:

Theorem 2.1. (Contraction mapping principle). Let $(X, d)$ be a complete metric space and $A: X \rightarrow X$ a contraction. Then $A$ is a Picard operator.

Theorem 2.2. (Characterization theorem). Let $(X, d)$ be a metric space and $A: X \rightarrow X$ an operator. The operator $A$ is a weakly Picard operator if and only if there exists a partition of $X$, $X=\cup_{\lambda \in \Lambda} X_{\lambda}$, such that:

(i) $X_{\lambda} \in I(A)$;

(ii) $\left.A\right|_{X_{\lambda}}: X_{\lambda} \rightarrow X_{\lambda}$ is a Picard operator, for all $\lambda \in \Lambda$.

Fibre generalized contraction theorem is a fixed point theorem for operators on cartezian product. This theorem is useful for proving solution of operatorial equations to be differentiable and it is a generalization of a result given by Hirsch and Pugh in [5]. See also [12], [20], [21].

Theorem 2.3. (Fibre contraction theorem). Let $(X, d)$ be a metric space, $(Y, \rho)$ be a complete metric space and $T: X \times Y \rightarrow X \times Y$. We suppose that:

(i) $T(x, y)=\left(T_{1}(x), T_{2}(x, y)\right)$, for all $x \in X, y \in Y$;

(ii) $T_{1}: X \rightarrow X$ is a weakly Picard operator;

(iii) there exists $c \in] 0,1[$, such that

$$
\rho\left(T_{2}\left(x, y_{1}\right), T_{2}\left(x, y_{2}\right)\right) \leq c \rho\left(y_{1}, y_{2}\right),
$$

for all $x \in X, y_{1}, y_{2} \in Y$.

Then the operator $T$ is a weakly Picard operator. Moreover, if $T_{1}$ is a Picard operator, then $T$ is a Picard operator.

We will use the previous result to study the differentiability with respect to parameter $\lambda$ for the solution of our equation.

\section{CAUCHY PROBLEM: EXISTENCE AND UNIQUENESS}

Let $\mathbf{K}:=\mathbf{R}$ or $\mathbf{C}, 0<\lambda<1$ and $f \in C\left([0, b] \times \mathbf{K}^{3}, \mathbf{K}\right)$.

We consider the following Cauchy problem:

$$
y^{\prime}(x)=f\left(x, y(x), y^{\prime}(x), y(\lambda x)\right), x \in[0, b],
$$

$$
y(0)=y_{0},
$$

where $y_{0} \in \mathbf{K}$. 
The problem (3.1)+(3.2) is equivalent to the following:

$$
\left\{\begin{array}{c}
y^{\prime}(x)=v(x) \\
v(x)=f\left(x, y_{0}+\int_{0}^{x} v(s) d s, v(x), y_{0}+\int_{0}^{\lambda x} v(s) d s\right) \\
y(0)=y_{0},
\end{array}\right.
$$

or

$$
\left\{\begin{array}{c}
y(x)=y_{0}+\int_{0}^{x} v(s) d s, x \in[0, b] \\
v(x)=f\left(x, y_{0}+\int_{0}^{x} v(s) d s, v(x), y_{0}+\int_{0}^{\lambda x} v(s) d s\right), \\
x \in[0, b] .
\end{array}\right.
$$

Let $T: C([0, b], \mathbf{K}) \rightarrow C([0, b], \mathbf{K})$ be the operator defined by

$$
(T(v))(x):=f\left(x, y_{0}+\int_{0}^{x} v(s) d s, v(x), y_{0}+\int_{0}^{\lambda x} v(s) d s\right) .
$$

So we obtain a fixed point problem

$$
v(x)=(T(v))(x), v \in C([0, b], \mathbf{K}) .
$$

The problem (3.1)+(3.2) has a unique solution if and only if the problem (3.5) has a unique solution, $v^{*} \in C([0, b], \mathbf{K})$.

Consequently, in our paper, we will study the fixed point problem (3.5).

By using Contraction principle we give an existence and uniqueness theorem.

Theorem 3.4. We suppose that there exist $L_{1}>0,0<L_{2}<1, L_{3}>0$ such that

$$
\left|f\left(x, u_{1}, u_{2}, u_{3}\right)-f\left(x, u_{4}, u_{5}, u_{6}\right)\right| \leq L_{1}\left|u_{1}-u_{4}\right|+L_{2}\left|u_{2}-u_{5}\right|+L_{3}\left|u_{3}-u_{6}\right|
$$

for all $x \in[0, b]$ and all $u_{k} \in K, k=\overline{1,6}$.

Then

(a) the problem (3.5) has a unique solution $v^{*} \in C([0, b], K)$;

(b) for all $v_{0} \in C([0, b], K)$ the sequence $\left(v_{n}\right)_{n \in \mathbb{N}}$ defined by

$$
v_{n+1}(x):=f\left(x, y_{0}+\int_{0}^{x} v_{n}(s) d s, v_{n}(x), y_{0}+\int_{0}^{\lambda x} v_{n}(s) d s\right),
$$

converges uniformly to $v^{*}$ on $[0, b]$.

Proof. Let $\|\cdot\|_{B}$ be a Bielecki norm on $C([0, b], \mathbf{K})$ defined by

$$
\|v\|_{B}=\max _{x \in[0, b]}|v(x)| e^{-\tau x}, \text { where } \tau>0 .
$$

Consider the above operator $T:\left(C([0, b], \mathbf{K}),\|\cdot\|_{B}\right) \rightarrow\left(C([0, b], \mathbf{K}),\|\cdot\|_{B}\right)$.

We have

$$
\begin{gathered}
|(T(v))(x)-(T(w))(x)| \leq L_{1} \int_{0}^{x}|v(s)-w(s)| d s+ \\
\quad+L_{2}|v(x)-w(x)|+L_{3} \int_{0}^{\lambda x}|v(s)-w(s)| d s \leq
\end{gathered}
$$




$$
\begin{gathered}
\leq L_{1} \int_{0}^{x}|v(s)-w(s)| e^{-\tau s} e^{\tau s} d s+L_{2}|v(x)-w(x)| e^{-\tau x} e^{\tau x}+ \\
+L_{3} \int_{0}^{x} \mid v(s)-w(s)\left\|e^{-\tau s} e^{\tau s} d s \leq \frac{L_{1}+L_{3}}{\tau}\left(e^{\tau x}-1\right)\right\| v-w \|_{B}+ \\
\quad+L_{2}\|v-w\|_{B} e^{\tau x} \leq\left(\frac{L_{1}+L_{3}}{\tau}+L_{2}\right)\|v-w\|_{B} e^{\tau x},
\end{gathered}
$$

for all $x \in[0, b]$.

Therefore,

$$
|(T(v))(x)-(T(w))(x)| e^{-\tau x} \leq\left(\frac{L_{1}+L_{3}}{\tau}+L_{2}\right)|| v-w \|_{B},
$$

for all $x \in[0, b]$.

This implies that

$$
\|T(v)-T(w)\|_{B} \leq\left(\frac{L_{1}+L_{3}}{\tau}+L_{2}\right)\|v-w\|_{B},
$$

for all $v, w \in C([0, b], \mathbf{K})$.

We can choose $\tau$ large enough such that $\frac{L_{1}+L_{3}}{\tau}+L_{2}<1$.

By applying Contraction principle we obtain $(a)$ and $(b)$.

Remark 3.1. Let us consider the operator

$$
A: C([0, b], \mathbf{K}) \times C([0, b], \mathbf{K}) \rightarrow C([0, b], \mathbf{K}) \times C([0, b], \mathbf{K})
$$

defined by

$$
\left.A(y, v)(x):=\left(y(0)+\int_{0}^{x} v(s) d s, f\left(x, y(0)+\int_{0}^{x} v(s) d s\right), v(x), y(0)+\int_{0}^{\lambda x} v(s) d s\right)\right) .
$$

From the Theorem 3.4. it is clear that the operator $A$, in the conditions of Theorem 3.4., is a weakly Picard operator. Indeed, let for $y_{0} \in \mathbf{K}$

$$
X_{y_{0}}:=\left\{y \in C([0, b], \mathbf{K}) \mid y(0)=y_{0}\right\}
$$

Then

$$
C([0, b], \mathbf{K}) \times C([0, b], \mathbf{K})=\bigcup_{y_{0} \in \mathbf{K}}\left(X_{y_{0}} \times C([0, b], \mathbf{K})\right)
$$

is an invariant partition of $C([0, b], \mathbf{K}) \times C([0, b], \mathbf{K})$, i.e.,

$$
A\left(X_{y_{0}} \times C([0, b], \mathbf{K})\right) \subset X_{y_{0}} \times C([0, b], \mathbf{K}), \text { for all } y_{0} \in \mathbf{K} .
$$

From a similar proof as in Theorem 3.4. we have that $A_{\mid X_{y_{0}} \times C([0, b], \mathbf{K})}$ is a Picard operator for each $y_{0} \in \mathbf{K}$. So, from Theorem 2.2., the operator $A$ is a weakly Picard operator.

From the definition of operator $A$ we have that:

- if $y$ is a solution of the equation 3.1., then $\left(y, y^{\prime}\right) \in F_{A}$;

- if $(y, v) \in F_{A}$, then $y$ is a solution of (3.1). 


\section{DATA DEPENDENCE}

By using Fibre contraction theorem we give a data dependence theorem for the solution of the following equation:

$$
v(x, \lambda)=(T(v))(x, \lambda), \quad v \in C([0, b] \times] 0,1[, \mathbf{K}) .
$$

We have

Theorem 4.5. We suppose that:

(i) $f(x, \cdot, \cdot, \cdot) \in C^{1}\left(\mathbf{K}^{3}\right)$, for all $x \in[0, b]$;

(ii) there exist $M_{k}>0, k=\overline{1,3}$, such that

$$
\left|\frac{\partial f}{\partial u_{k}}\left(x, u_{1}, u_{2}, u_{3}\right)\right| \leq M_{k}, k=\overline{1,3},
$$

for all $x \in[0, b]$ and all $u_{k} \in \mathbf{K}, k=\overline{1,3}$;

(iii) $0<M_{2}<1$.

Then

(a) the equation (4.6) has a unique solution $v^{*} \in C([0, b] \times] 0,1[, \mathbf{K})$;

(b) for all $v_{0} \in C([0, b] \times] 0,1[, \mathbf{K})$ the sequence $\left(v_{n}\right)_{n \in \mathbb{N}}$ defined by

$$
v_{n+1}(x, \lambda):=f\left(x, y_{0}+\int_{0}^{x} v_{n}(s, \lambda) d s, v_{n}(x, \lambda), y_{0}+\int_{0}^{\lambda x} v_{n}(s, \lambda) d s\right)
$$

converges uniformly to $v^{*}$ on each compact of $\left.[0, b] \times\right] 0,1[$;

(c) the sequence $\left(w_{n}\right)_{n \in \mathbb{N}}$ defined by

$$
\begin{gathered}
w_{n+1}(x, \lambda):= \\
=\frac{\partial f}{\partial u_{1}}\left(x, y_{0}+\int_{0}^{x} v_{n}(s, \lambda) d s, v_{n}(x, \lambda), y_{0}+\int_{0}^{\lambda x} v_{n}(s, \lambda) d s\right) \int_{0}^{x} w_{n}(s, \lambda) d s+ \\
+\frac{\partial f}{\partial u_{2}}\left(x, y_{0}+\int_{0}^{x} v_{n}(s, \lambda) d s, v_{n}(x, \lambda), y_{0}+\int_{0}^{\lambda} v_{n}(s, \lambda) d s\right) w_{n}(x, \lambda)+ \\
+\frac{\partial f}{\partial u_{3}}\left(x, y_{0}+\int_{0}^{x} v_{n}(s, \lambda) d s, v_{n}(x, \lambda), y_{0}+\int_{0}^{\lambda x} v_{n}(s, \lambda) d s\right) . \\
\cdot\left(\int_{0}^{\lambda x} w_{n}(s, \lambda) d s+x v_{n}(\lambda x, \lambda)\right),
\end{gathered}
$$

where $w_{0}=\frac{\partial v_{0}}{\partial \lambda}$, converges uniformly to $\frac{\partial v^{*}}{\partial \lambda}$ on each compact of $\left.[0, b] \times\right] 0,1[$.

Proof. For $0<\lambda_{1}<\lambda_{2}<1$, we denote $X=\left(C\left([0, b] \times\left[\lambda_{1}, \lambda_{2}\right], \mathbf{K}\right),\|\cdot\|_{\tau}\right)$, where

$$
\|v\|_{\tau}=\max _{\substack{x \in[0, b] \\ \lambda \in\left[\lambda_{1}, \lambda_{2}\right]}}|v(x, \lambda)| e^{-\tau x}
$$

and $\tau>0$.

Consider the operator $S_{1}: X \rightarrow X$, defined by

$$
\left(S_{1}(v)\right)(x, \lambda):=f\left(x, y_{0}+\int_{0}^{x} v(s, \lambda) d s, v(x, \lambda), y_{0}+\int_{0}^{\lambda x} v(s, \lambda) d s\right) .
$$


The operator $S_{1}$ is a Lipchitz operator with the constant

$$
L_{S_{1}}=\frac{M_{1}+M_{3}}{\tau}+M_{2} \text {. }
$$

Because of condition (iii), and by choosing $\tau$ large enough we have that $L_{S_{1}}<1$.

By applying Contraction principle to the operator $S_{1}$ we obtain (a) and (b).

Let us prove that there exists $\frac{\partial v^{*}}{\partial \lambda}$ and

$$
\frac{\partial v^{*}}{\partial \lambda}(x, \cdot) \in C\left(\left[\lambda_{1}, \lambda_{2}\right], \mathbf{K}\right), \text { for all } x \in[0, b] .
$$

We have

$$
v^{*}(x, \lambda)=f\left(x, y_{0}+\int_{0}^{x} v^{*}(s, \lambda) d s, v^{*}(x, \lambda), y_{0}+\int_{0}^{\lambda x} v^{*}(s, \lambda) d s\right) .
$$

If we suppose that there exists $\frac{\partial v^{*}}{\partial \lambda}$, then from (4.7) we obtain

$$
\begin{aligned}
\frac{\partial v^{*}}{\partial \lambda}(x, \lambda)=\frac{\partial f}{\partial u_{1}} & \left(x, y_{0}+\int_{0}^{x} v^{*}(s, \lambda) d s, v^{*}(x, \lambda), y_{0}+\int_{0}^{\lambda x} v^{*}(s, \lambda) d s\right) \int_{0}^{x} \frac{\partial v^{*}}{\partial \lambda}(s, \lambda) d s+ \\
+ & \frac{\partial f}{\partial u_{2}}\left(x, y_{0}+\int_{0}^{x} v^{*}(s, \lambda) d s, v^{*}(x, \lambda), y_{0}+\int_{0}^{*} v^{*}(s, \lambda) d s\right) \frac{\partial v^{*}}{\partial \lambda}(x, \lambda)+ \\
+ & \frac{\partial f}{\partial u_{3}}\left(x, y_{0}+\int_{0}^{x} v^{*}(s, \lambda) d s, v^{*}(x, \lambda), y_{0}+\int_{0}^{\lambda x} v^{*}(s, \lambda) d s\right) . \\
& \cdot\left(\int_{0}^{\lambda x} \frac{\partial v^{*}}{\partial \lambda}(s, \lambda) d s+x v^{*}(\lambda x, \lambda)\right) .
\end{aligned}
$$

The previous relationship suggests us to consider the operator $S_{2}: X \times X \rightarrow X$, defined by

$$
\begin{gathered}
\left(S_{2}(v, y)\right)(x, \lambda):= \\
=\frac{\partial f}{\partial u_{1}}\left(x, y_{0}+\int_{0}^{x} v(s, \lambda) d s, v(x, \lambda), y_{0}+\int_{0}^{\lambda x} v(s, \lambda) d s\right) \int_{0}^{x} y(s, \lambda) d s+ \\
+\frac{\partial f}{\partial u_{2}}\left(x, y_{0}+\int_{0}^{x} v(s, \lambda) d s, v(x, \lambda), y_{0}+\int_{0}^{\lambda x} v(s, \lambda) d s\right) y(x, \lambda)+ \\
+\frac{\partial f}{\partial u_{3}}\left(x, y_{0}+\int_{0}^{x} v(s, \lambda) d s, v(x, \lambda), y_{0}+\int_{0}^{\lambda x} v(s, \lambda) d s\right) . \\
\cdot\left(\int_{0}^{\lambda x} y(s, \lambda) d s+x v(\lambda x, \lambda)\right) .
\end{gathered}
$$

By using (i) and(ii) we obtain that

$$
\left\|S_{2}\left(v, y_{1}\right)-S_{2}\left(v, y_{2}\right)\right\|_{\tau} \leq\left(\frac{M_{1}+M_{3}}{\tau}+M_{2}\right)\left\|y_{1}-y_{2}\right\|_{\tau}
$$


for all $y_{1}, y_{2} \in X$.

Because of condition (iii), and by choosing $\tau$ large enough, we have that $S_{2}$ is a contraction with respect to the second argument.

If we take the operator $S: X \times X \rightarrow X \times X, S=\left(S_{1}, S_{2}\right)$ then we are in the conditions of Fibre contraction theorem. Let $\left(v^{*}, w^{*}\right)$ the unique fixed point of the operator $S$.

If we take $v_{0}=0, w_{0}=0$ then $w_{1}=\frac{\partial v_{1}}{\partial \lambda}$.

By mathematical induction method we can prove that $w_{n}=\frac{\partial v_{n}}{\partial \lambda}$.

Thus $\left(v_{n}\right)_{n \in \mathbb{N}}$ converges uniformly to $v^{*}$ and $\left(\frac{\partial v_{n}}{\partial \lambda}\right)_{n \in \mathbb{N}^{*}}$ converges uniformly to $w^{*}$. By using a Weiestrass argument we obtain that $\frac{\partial v^{*}}{\partial \lambda}$ exists and $\frac{\partial v^{*}}{\partial \lambda}=w^{*}$, and $w^{*}$ is a continuous function.

So, we have (c).

\section{REFERENCES}

[1] Azbelev, N. V., Maksimov, V. P. and Rahmatulina, L. F., Introduction to Functional - Differential Equations Theory, MIR, Moscow, 1991 (In Russian)

[2] Burton, T. A., Stability by Fixed Point Theory for Functional Differential Equations, Dover Publ., Mineola, 2006

[3] Guo, D., Lakshmikantham, V. and Liu, X., Nonlinear Integral Equations in Abstract Spaces, Kluwer Acad. Publ., Dordrecht, 1996

[4] Hale, J. K. and Sjoerd, M. Verduyn Lunel, Introduction to Functional - Differential Equations, Springer Verlag, New York, 1993

[5] Hirsch, M. W. and Pugh, C. C., Stable manifolds and hyperbolic sets, Proc. Symp. in Pure Math., 14 (1970), 133-163

[6] Ionescu, D. V., L'application de la méthode des approximation successive à l'integration numérique des l'equations differentielles, Bull. Math. Soc. Sci. Math. Roumanie, 51 (1959), No. 3, 423 -431

[7] Iserles, A., On the generalized pantograph functional - differential equation, European J. Appl. Math., 4 (1992), $1-38$

[8] Mureşan, V., Differential Equation with Affine Modification of the Argument, Transilvania Press, Cluj-Napoca, 1997 (In Romanian)

[9] Mureşan, V., Functional - Integral Equations, Ed. Mediamira, Cluj - Napoca, 2003

[10] Ockendon, J. R., Differential equations and industry, The Math. Scientist, 5 (1980), No. 1, 1-12

[11] Rus, I. A., Weakly Picard mappings, Comment. Math. Univ. Carolin., 34 (1993), No. 4, 769-773

[12] Rus, I. A., A fiber generalized contraction theorem and applications, Mathematica, 41(64) (1999), No. 1, 85-90

[13] Rus, I. A., A class of nonlinear functional-integral equations, via weakly Picard operators , Ann. Univ. Craiova, Ser. Mat.-Inform., 28 (2001), 10-15

[14] Rus, I. A., Picard operators and applications, Sci. Math. Jpn., 58 (2003), No. 1, 191-219

[15] Rus, I. A., Some nonlinear functional differential and integral equations, via weakly Picard operators theory: a survey, Carpathian J. Math., 26 (2010), No. 2, 230-258

[16] Rus, I. A., Some variants of contraction principles in the case of operators with Volterra property: step by step contraction principle, Advances in the Theory of Nonlinear Anal. and its Appl., 3 (2019), No. 3, 111-120

[17] Rus, I. A., Petruşel A. and Şerban M. A., Fibre Picard operators on gauge spaces and applications, Z. Anal. Anwend., 27 (2008), No. 4, 407-423

[18] Rus, I. A. and Şerban M. A., Basic problem of the metric fixed point theory and the relevance of a metric fixed point theorem, Carpathian J. Math., 29(2013), No. 2, 239-258

[19] Szep, G., On an integral equation with deviating argument, Seminar on Fixed Point Theory Cluj-Napoca, 1 (2000), 103-108

[20] Şerban, M. A., Fixed Point Theory for Operators defined on Cartezian Product, Presa Univ. Clujeană, ClujNapoca, 2002 (In Romanian)

[21] Şerban, M. A., Rus, I. A. and Petruşel, A., A class of abstract Volterra equations, via weakly Picard operators' technique , Math. Inequal. Appl., 13 (2010), No. 2, 255-269 
Department of Statistics, Forecasts and Mathematics

BABEŞ-BOLYAi UNIVERSITY OF ClUJ-NAPOCA

T. Mihaly StreEt No. 58-60, 400591, Cluj-NAPOCA, Romania

Email address: anton.muresan@econ. ubbcluj.ro

DEPARTMENT OF MATHEMATICS

TECHNICAL UNIVERSITY OF CLUJ-NAPOCA

Memorandumului StreEt No. 28, 400114, Cluj-NAPOCA, Romania

Email address: vmuresan@math.utcluj.ro 